\title{
Associations between left ventricular structure and function with cardiorespiratory fitness and body composition in individuals with cervical and upper thoracic spinal cord injury
}

\author{
Abdullah A. Alrashidi ${ }^{1,2,3} \cdot$ Shane J. T. Balthazaar $\mathbb{D}^{1,2} \cdot$ Katharine D. Currie $^{1,4} \cdot$ Tom E. Nightingale $\mathbb{D}^{1,5}$. \\ Andrei V. Krassioukov (iD) ${ }^{1,6,7}$
}

Received: 28 July 2020 / Revised: 10 November 2020 / Accepted: 11 November 2020 / Published online: 7 December 2020

Crown 2020. This article is published with open access

\begin{abstract}
Study design Cross-sectional.

Objective It is known that left ventricular mass (LVM) and cardiorespiratory fitness (CRF) are associated to fat-free mass (FFM). It is unknown if these factors associated with left ventricular (LV) structure and function outcomes in individuals with spinal cord injury (SCI).

Setting University-based laboratory.Vancouver, BC, Canada.

Methods Thirty-two individuals (aged $40 \pm 11$ years) with chronic, motor-complete SCI between the fourth cervical and sixth thoracic levels were recruited. Echocardiographic LV parameters and body composition were assessed at rest, as per the recommended guidelines for each technique. CRF was assessed during an incremental arm-cycle exercise test until volitional fatigue. The appropriate bivariate correlation coefficients [i.e., Pearson's $(r)$ and Spearman's rank $\left(R_{s}\right)$ ] tests were used for normal and non-normal distributed variables, respectively.

Results LV structure and function parameters were not associated with the indexed peak oxygen consumption $\left(\dot{V}_{2} \mathrm{O}_{2 \mathrm{pak}}\right)$ [i.e., relative to body weight or FFM] $\left(R_{s}\right.$ values ranged from -0.168 to 0.134 , all $P$ values $\left.>0.223\right)$. The association between peak oxygen pulse and the resting echocardiographic-obtained SV was medium sized $\left(R_{S}=0.331, P=0.069\right)$. The LVM associations with FFM and fat mass (FM) were large and small $(r=0.614, P<0.001$ and $r=0.266, P=0.141$, respectively). Associations of absolute $\dot{\mathrm{V}} \mathrm{O}_{2 \text { peak }}$ were medium- positive with FFM $\left(R_{s}=0.414, P=0.021\right)$ but negative with FM $\left(R_{s}=-0.332, P=0.068\right)$.

Conclusion LV parameters measured at rest are not associated with $\dot{\mathrm{VO}}_{2 \text { peak }}$ in individuals with cervical and upper-thoracic SCI. Given the observed associations between LVM and $\mathrm{VO}_{2 \text { peak }}$ with FFM, future studies may consider utilizing FFM for indexing cardiovascular measures following SCI.
\end{abstract}

\section{Introduction}

It is well-established in non-injured individuals that higher levels of cardiorespiratory fitness (CRF) are associated with a reduced risk of cardiovascular disease (CVD), all-cause

Andrei V. Krassioukov

krassioukov@icord.org

1 International Collaboration On Repair Discoveries, University of British Columbia, Vancouver, BC, Canada

2 Department of Experimental Medicine, University of British Columbia, Vancouver, BC, Canada

3 Department of Physical Therapy, King Fahad Medical City, Riyadh, Saudi Arabia mortality, and other inactivity-related chronic diseases [1]. Individuals with spinal cord injury (SCI), are known to have a reduced CRF, which is in part due to increased levels of physical inactivity following SCI [2, 3]. Furthermore, individuals with motor-complete, cervical injuries tend to

4 Department of Kinesiology, Michigan State University, East Lansing, MI, USA

5 School of Sport, Exercise and Rehabilitation Sciences, University of Birmingham, Edgbaston, Birmingham, UK

6 Division of Physical Medicine and Rehabilitation, University of British Columbia, Vancouver, BC, Canada

7 G.F. Strong Rehabilitation Centre, Vancouver, BC, Canada 
display lower levels of CRF [2, 4]. Collectively, this increases the risk of CVD events and cardiometabolic dysfunction, all of which negatively impact independence and quality of life in this population [5].

In the non-injured population, prolonged inactivity and decreased hemodynamic load, even with the absence of apparent CVD, have been linked with a smaller cardiac cavity size and mass [6]. A recent meta-analysis revealed a reduction in left ventricular mass (LVM), reduced stroke volume (SV), and altered diastolic function in individuals with SCI compared to the non-injured population [7]. Alongside reduced CRF and lower hemodynamic load, injuries at and above the sixth thoracic $(\geq \mathrm{T} 6)$ spinal segment not only diminishes sympathetic control to the peripheral vasculature but may also compromise sympathetic drive to the heart (extends from between the T1-T5 spinal segments) [8]. Left ventricular (LV) dysfunction and perturbed autonomic function [9] may limit cardiac output ( $\dot{\mathrm{Q}})$ and $\mathrm{SV}$, in turn reducing the maximal attainable level of CRF [i.e., lower peak oxygen consumption $\left.\left(\mathrm{V}_{2 \text { peak }}\right)\right]$. CVD events are elevated in individuals with SCI [10]; therefore, assessing $\mathrm{LV}$ dysfunction in conjunction with reduced CRF is highly relevant, given studies have shown that both are linked with increased risks of CVD morbidity and mortality in non-injured and select clinical populations $[1,11,12]$.

The association between resting echocardiographic LV parameters and CRF has been investigated in non-injured individuals and certain clinical populations. The results of these studies were controversial with some reporting there is an association [13-15] while other reported that this association does not exist $[16,17]$. The association between CRF and LV diastolic function is more evident compared to that with systolic function [14, 15, 18]. One mechanism that may explain the association between LV diastolic function and CRF is the capability of LV to generate maximal $\dot{Q}$ via the maintenance of adequate $\mathrm{LV}$ filling pressure [13].

Cardiac dimensions are very closely associated with body dimensions (e.g., body mass and height). Thus, for the accurate quantification of cardiac dimensions and functional measures, as well as for inter- and intra-group comparison, the effect of body size should be partitioned out. Previous studies involving non-injured individuals have shown that LVM is closely related to body size and composition, as well as demonstrating that fat-free mass (FFM) is strongly associated with and a reliable predictor of LVM compared with other body dimensions [e.g., body surface area (BSA), body mass, height] $[19,20]$. While it is common to report LVM indexed to BSA [21], such indexing might underestimate LVM measurements and fail to identify individuals with obesity and LVM abnormalities [22]. Individuals with $\mathrm{SCI}$, as a result of paralysis, are at increased risk of obesity and altered body composition compared to non-injured individuals [23]. Thus, indexing LVM to BSA may pose the same problem with this population.

The purpose of this study was to investigate the associations between LV structure and function with CRF. We hypothesized that those with a higher $\mathrm{V}_{2 \text { peak }}$ would have more favorable resting LV parameters. Due to the observed association between LVM and body composition in the non-injured population, we also explored this association in our cohort (i.e., cervical and upper-thoracic SCI).

\section{Methods}

\section{Participants}

These data were collected from a larger prospective, multicenter, randomized clinical trial [The Cardiovascular Health/ Outcomes: Improvements Created by Exercise and Education in SCI (CHOICES)] conducted at the University of British Columbia, with the registration number (NCT01718977) on ClinicalTrial.gov, November 1, 2012, the study protocol of which has been previously published [24]. All participants provided informed consent and all measurements were collected as part of the baseline assessments of CHOICES (between April 2013 and August 2017) before the commencement of the training interventions. Leisure time physical activity was captured using the recently validated Leisure Time Physical Activity Questionnaire for People with SCI (LTPAQ-SCI) [25]. All participants arrived at the laboratory following a minimum $4 \mathrm{~h}$ fast and avoided vigorous physical activity (PA) for the preceding $24 \mathrm{~h}$. This study included the data of participants who were recruited from the International Collaboration On Repair Discoveries (ICORD), Vancouver, BC, Canada, in which echocardiography was performed. The inclusion criteria were as follows: Adults 18-60 years of age with chronic [time since injury (TSI) $>1$ year], motor-complete [American Spinal Injury Association Impairment Scale (AIS) A-B)], traumatic SCI between the fourth cervical $(\mathrm{C} 4)$ and $\mathrm{T} 6$ segments. The neurological level of injury (NLI) and AIS were assessed and determined using the International Standards for Neurological Classification of SCI [26]. Exclusion criteria included any medical history or symptoms of CVD, major trauma/surgery in the last 6 months, an unstable medical/psychological condition, or any cognitive dysfunction that would be a barrier to understanding English.

\section{Outcome measures}

\section{LV structure and function}

All participants underwent non-invasive trans-thoracic echocardiography (Vivid 7, GE Healthcare, Horton, 
Norway) in the left lateral decubitus position, as per the recommendations of the American Society for Echocardiography [27]. Images were collected and analyzed offline via dedicated software (EchoPAC; GE Healthcare, Horton, Norway). The average of three cardiac cycles was used to determine LV structure and global functions. Measures of LV structure at the end-diastole and endsystole were reported from the parasternal long-axis views. LVM was calculated and indexed to BSA according to a well-established formula [28] (i.e., the Devereux method). Volumetric measurements and systolic functions were derived from the apical four-chamber biplane views using the modified Simpson method. $\dot{Q}$ was calculated as the product of SV and heart rate (HR). The LV diastolic function comprised early peak mitral annular septal tissue velocity (E'); early (E) and late (A) peak transmitral flow, and the early-to-late transmitral filling velocity (E/A) ratio, which were analyzed using the pulsed-wave Doppler. The E/E' ratio, a validated estimate of LV filling pressure, was calculated. Additional diastolic indices included deceleration time, defined as the maximum downward slope of the early peak flow rate, and isovolumetric relaxation time, defined as the time interval from the aortic valve closing to the mitral valve opening, as determined on the spectral Doppler trace [27]. Intraclass correlation coefficients (ICCs) were performed on ten randomly selected participants from our cohort, calculating all echocardiography outcomes across three cardiac cycles. ICCs showed a high degree of reliability (mean range: 0.918 to 0.993 ) with $95 \%$ confidence intervals of $0.218-0.998$.

\section{Cardiorespiratory fitness}

An incremental arm-crank exercise test on an electrically braked arm-crank ergometer (Lode BV, Groningen, The Netherlands), done until volitional exhaustion was performed to determine the peak parameters. HR was recorded continuously using a chest-strap HR monitor (T31; Polar Electro Inc., Woodbury, NY, USA). Respiratory gases were collected using a metabolic cart (Parvomedics Truemax 2400, Sandy, UT, USA) calibrated immediately before each test. Participants performed the test while sitting in their wheelchair and were asked to empty their bladder prior to the test to avoid the possible development of autonomic dysreflexia. After two minutes of resting, the test protocol began with a warm-up with no resistance for two minutes and then continued with one-minute stages, where resistance was increased by $5-10$ Watts per stage for participants with cervical and upper-thoracic NLI, respectively [29]. The participants were instructed to maintain a cadence of 50 revolutions per minute (rpm) throughout the test. The test continued with verbal encouragement until volitional exhaustion was experienced or the cadence dropped below
$30 \mathrm{rpm}$. The test ended with a two-minute cool-down period with no resistance. Peak gas exchange values were collected, and the highest $20 \mathrm{~s}$ average was taken as the peak value. $\dot{\mathrm{VO}}_{2 \text { peak }}$ was normalized to body mass and FFM. Peak oxygen pulse $\left(\mathrm{O}_{2 \text { pulse }}\right)$ was calculated as a ratio between $\dot{\mathrm{VO}}_{2 \text { peak }}$ and $\mathrm{HR}_{\text {peak }}$.

\section{Body composition}

Body composition was measured using Dual-energy X-ray absorptiometry (DXA) (Hologic 4500A or W densitometer, Waltham, Massachusetts, USA). Outcomes were reported as per the International Society for Clinical Densitometry guidelines and as detailed previously in the study protocol [24]. The study coordinator assisted the transfer to the DXA plinth through the use of a sliding board, as necessary. An expert DXA technologist checked the participant's posture while on the plinth and ensured that all limbs were aligned and without any rotation or shifting of the pelvis and/or trunk. For the purpose of this study, we only reported FFM and fat mass (FM) data in our analysis.

\section{Statistical analyses}

All statistical analyses were performed using the Statistical Package for the Social Sciences (SPSS, version 24; IBM Corporation, Armonk, USA) and GraphPad Prism (version 6). All data were visually inspected and analyzed for normality using a Q-Q plot and Shapiro-Wilk test, respectively. To investigate the association between CRF, LV parameters, and body composition, bivariate correlation coefficient [i.e., Pearson's $(r)$ or Spearman's rank $\left(R_{s}\right)$ ] tests were used for normal and non-normal distributed variables, respectively. We also ran partial correlation coefficients controlling for pre-determined covariates (i.e., age, NLI, TSI). The NLI was converted into a continuous variable, where $\mathrm{C} 4=1$, and $\mathrm{C} 5=2$, and so on until $\mathrm{T} 6=11$. The magnitude of each correlation was interpreted using the following: small $(r>0.1)$, medium $(r>0.3)$, large $(r>0.5)$, and very large $(r>0.7)$. Univariate (simple) regression $\left(R^{2}\right)$ was used where appropriate (i.e., predicting LVM from FFM).

\section{Results}

A total of 32 participants were included. Participants' demographics, injury characteristics, peak CRF, body composition parameters and level of leisure time PA are presented in Table 1. Of the study participants, males and individuals with cervical injury accounted for $75 \%$ and $66 \%$, respectively, and the majority were classified as AIS A $(72 \%)$. None of the resting echocardiographic LV 
Table 1 Participant characteristics.

\begin{tabular}{|c|c|c|}
\hline & Cervical $N=21$ & Thoracic $N=11$ \\
\hline \multicolumn{3}{|l|}{ Age $(y)$} \\
\hline & $41 \pm 11$ & $37 \pm 10$ \\
\hline \multicolumn{3}{|l|}{ Sex } \\
\hline Male/female & $15 / 6$ & $9 / 2$ \\
\hline \multicolumn{3}{|l|}{ AIS } \\
\hline $\mathrm{A} / \mathrm{B}^{\mathrm{a}}$ & $12 / 9$ & $11 / 0$ \\
\hline Time since injury (yrs.) & $10(15)$ & $8(21)$ \\
\hline Height $(\mathrm{cm})$ & $178 \pm 11$ & $176 \pm 8$ \\
\hline Mass (kg) & $76 \pm 24$ & $76 \pm 14$ \\
\hline BMI $\left(\mathrm{kg} / \mathrm{m}^{2}\right)$ & $23.7 \pm 3.3$ & $24.6 \pm 3.4$ \\
\hline $\operatorname{BSA}\left(m^{2}\right)$ & $1.9 \pm 0.3$ & $1.9 \pm 0.1$ \\
\hline \multicolumn{3}{|l|}{ Peak cardiorespiratory fitness } \\
\hline$\dot{\mathrm{VO}}_{2}(\mathrm{~mL} / \mathrm{kg} / \mathrm{min})^{\mathrm{a}}$ & $8.17(3.71)$ & $14.41(5.69)$ \\
\hline$\dot{\mathrm{V}} \mathrm{O}_{2}\left(\mathrm{~mL} / \mathrm{kg}_{\mathrm{FFM}} / \mathrm{min}\right)^{\mathrm{a}}$ & $13.55(6.14)$ & $19.77(8.64)$ \\
\hline$\dot{\mathrm{V}} \mathrm{O}_{2}(\mathrm{~L} / \mathrm{min})^{\mathrm{a}}$ & $0.69(0.36)$ & $1.04(0.42)$ \\
\hline $\mathrm{O}_{2 \text { pulse }}(\mathrm{mL} / \mathrm{beat})$ & $6.83 \pm 1.97$ & $7.65 \pm 2.08$ \\
\hline \multicolumn{3}{|l|}{ Body composition } \\
\hline FFM $(\mathrm{kg})$ & $48.5 \pm 11.8$ & $52.9 \pm 7.2$ \\
\hline $\mathrm{FM}(\mathrm{kg})$ & $25.2 \pm 7.7$ & $20.6 \pm 6.2$ \\
\hline FFMI $\left(\mathrm{kg} / \mathrm{m}^{2}\right)$ & $15.2 \pm 2.5$ & $17.2 \pm 1.6$ \\
\hline \multicolumn{3}{|l|}{ Physical activity } \\
\hline Mild LTPA (min/week) & $120(180)$ & $100(120)$ \\
\hline Moderate LTPA (min/week) & $68(240)$ & $105(240)$ \\
\hline Heavy LTPA (min/week) & $35(90)$ & $70(270)$ \\
\hline Total LTPA (min/week) & $300(505)$ & $315(627)$ \\
\hline
\end{tabular}

Data are mean \pm standard deviation for parametric variables (age, height, mass, BMI, BSA, $\mathrm{O}_{2 \text { pulse }}, \mathrm{FFM}, \mathrm{FM}$ and FFMI). Nonparametric variables (time since injury, $\dot{\mathrm{VO}}_{2}$ and LTPA) are presented as median (interquartile range). Categorical variables (sex and AIS) are presented as participant numbers.

AIS American Spinal Injury Association Impairment Scale, $B M I$ body mass index, $B S A$ body surface area, $B M I$ body mass index, $B S A$ body surface area, $\dot{V} O_{2}$ oxygen consumption, $F F M$ fat-free mass, $F M$ fat mass, FFMI fat-free mass index, LTPA leisure time physical activity. ${ }^{a}$ Indicates significant between-group differences at 0.05 (Chi-squared and Mann-Whitney U tests for AIS and $\dot{\mathrm{VO}}_{2 \text { peak }}$, respectively). structure and function parameters were associated with relative $\mathrm{VO}_{2 \text { peak }}$ (i.e., relative to body mass or FFM) (Table 2). The association between peak oxygen pulse $\left(\mathrm{O}_{2 \text { pulse }}\right)$, a surrogate of exercise $\mathrm{SV}$ that was obtained at peak CRF testing, was medium with resting echocardiographic-obtained SV $\left(R_{s}=0.331, P=0.069\right)$. Associations between absolute $\dot{\mathrm{V}} \mathrm{O}_{2 \text { peak }}$ with FFM were medium- positive $\left(R_{s}=0.420, P=0.017\right)$ but negative with FM $\left(R_{s}=-0.360, P=0.043\right)$. LVM associations with FFM and FM were large and small $(r=0.614, P<0.001$ and $r=$ $0.266, P=0.141$, respectively) (Fig. 1). The association between LVM and FFM remained large after partially controlling for age, NLI, and TSI $(r>0.607, P<0.001)$.

\section{Discussion}

The present study examined the associations between resting echocardiographic LV structure and function parameters and CRF, as well as exploring the associations between LVM and DXA-obtained body composition (i.e., FFM and FM) in individuals with cervical and upper-thoracic SCI. The results of this study show that none of the resting LV parameters (i.e., structure and function) are associated with $\dot{\mathrm{V}}{ }_{2 \text { peak }}$ (relative to body mass or FFM). There is a medium association between CRF test-obtained peak $\mathrm{O}_{2 \text { pulse }}$, a surrogate of exercise $\mathrm{SV}$, and resting echocardiographicobtained SV. Although LV parameters have been shown to be altered following SCI compared to non-injured individuals [7], these resting parameters may not necessarily correlate with reduced CRF levels. Our results show that LVM was positively associated with FFM in individuals with motor-complete (i.e., AIS A and B) and above midthoracic level ( $\geq \mathrm{T} 6)$ SCI.

Two large cross-sectional studies that recruited individuals referred for an echocardiogram, with no overt cardiac disease, showed that CRF measured in metabolic equivalents was strongly and inversely associated with LV diastolic dysfunction $[13,14]$. No associations were observed

Table 2 Bivariate association between CRF and resting LV parameters.

\begin{tabular}{|c|c|c|c|c|c|c|c|}
\hline & \multirow[b]{2}{*}{ Mean $\pm S D(C R F)$} & \multicolumn{6}{|c|}{ LV parameters } \\
\hline & & $\mathrm{SV}(\mathrm{mL})$ & $\dot{\mathrm{Q}}$ (L/min) & $\operatorname{LVM}(\mathrm{g})$ & E/A ratio & E/E’ septal & Deceleration time (ms) \\
\hline Mean $\pm S D(L V)$ & & $57.47 \pm 9.18$ & $3.59 \pm 0.87$ & $147.8 \pm 42.7$ & $1.59 \pm 0.59$ & $7.12 \pm 2.14$ & $221 \pm 75$ \\
\hline \multicolumn{8}{|l|}{ CRF } \\
\hline$\dot{\mathrm{V}} \mathrm{O}_{2 \text { peak }}(\mathrm{mL} / \mathrm{kg} / \mathrm{min})$ & $11.23 \pm 4.43$ & -0.047 & 0.088 & -0.225 & 0.050 & -0.024 & -0.076 \\
\hline$\dot{\mathrm{V}} \mathrm{O}_{2 \text { peak }}\left(\mathrm{mL} / \mathrm{kg}_{\mathrm{FFM}} / \mathrm{min}\right)$ & $17.35 \pm 4.43$ & -0.066 & -0.078 & -0.168 & 0.134 & -0.046 & -0.044 \\
\hline
\end{tabular}

$S V$ stroke volume, $\dot{Q}$ cardiac output, $L V M$ left ventricular mass, E/A ratio ratio of early-to-late transmitral. velocity, $E / E^{\prime}$ mitral peak E-wave to peak mitral annulus velocity ratio, $\dot{V} O_{2 p e a k}$ peak oxygen consumption. 

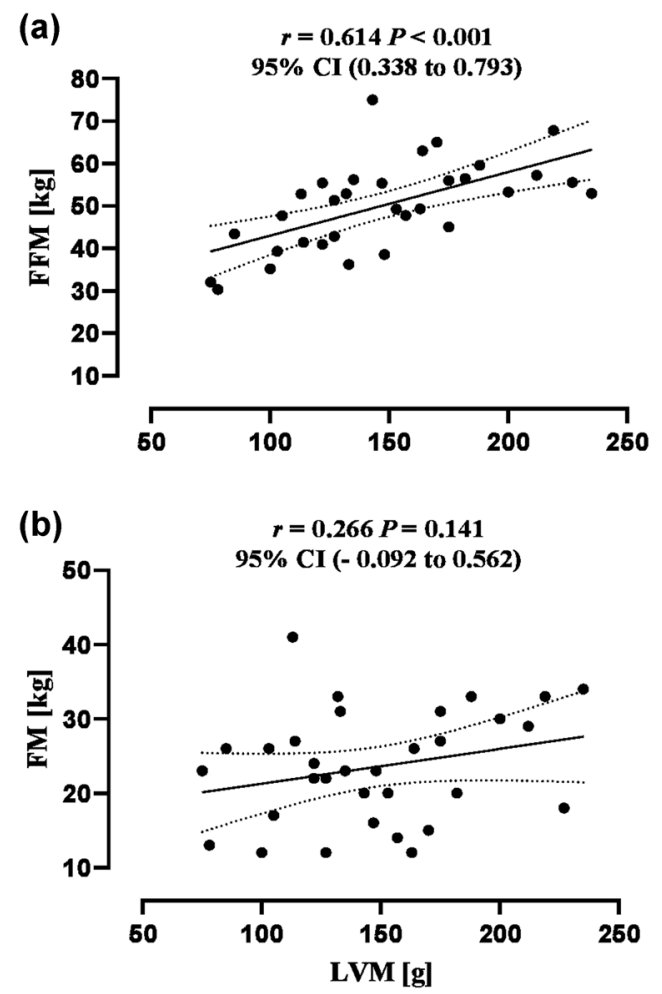

Fig. 1 Associations between left ventricular mass (LVM) with fat-free mass (FFM) and fat mass (FM). Association of LVM with FFM (a) and FM (b). Broken lines denote 95\% confidence intervals.

with systolic function. Likewise, the CARDIA study [15] comprising healthy, middle-age individuals found strong associations between CRF (i.e., treadmill time as a surrogate) and LV diastolic and systolic functions. However, the associations between CRF and diastolic parameters were higher compared with parameters of systolic function. In clinical populations, Franciosa et al [16]. conducted a study on individuals with congestive heart failure and showed that there were no associations between resting LV systolic function (i.e., $\dot{Q}, \mathrm{SV}$, and ejection fraction) and exercise duration. With the same clinical population, Szlachcic et al [17]. also found no association between $\mathrm{VO}_{2 \max }$ and cardiac function at rest, except for an inverse association with LV filling pressure (pulmonary capillary wedge pressure as a surrogate).

To the best of our knowledge, Maggioni et al. [30] was the only group that investigated this association between $\dot{\mathrm{V}} \mathrm{O}_{2 \text { peak }}$ and resting $\mathrm{LV}$ parameters in the SCI population. In this study, 17 males with paraplegia (i.e., T1-L3) were compared against non-injured individuals. In individuals with SCI, and independent of training status, they found that relative $\dot{\mathrm{VO}}_{2 \text { peak }}$ was positively associated $(r=0.36$ and $P<$ 0.05 ) with aortic flow velocity (a surrogate of SV). This association was documented when trained and untrained participants were combined and was reflected by a higher relative $\dot{\mathrm{V}} \mathrm{O}_{2 \text { peak }}$ compared to our cohort $(\sim 17.6 \mathrm{~mL} / \mathrm{kg} / \mathrm{min}$ vs. $11.2 \mathrm{~mL} / \mathrm{kg} / \mathrm{min}$ ). We used $\mathrm{O}_{2 \text { pulse, }}$ a surrogate of exercise $\mathrm{SV}$, and this demonstrated a medium-sized association with the resting echocardiographic-obtained SV. Participants in our study were motor-complete and had a higher NLI (C4-T6). The disparity in $\mathrm{VO}_{2 \text { peak }}$ between our results and theirs may be related the inclusion of different cohorts characterized by AIS and NLI or the different modalities used for peak exercise testing (wheelchair ergometer vs. arm ergometer). Besides the participants in the Maggioni et al. study having a greater degree of sympathetic innervation to splanchnic beds, wheelchair propulsion may increase venous return and eventually improve SV. This could be due to the movement mechanics possibly mimicking an abdominal binder [31]. Further discrepancies stem from the difference in echocardiography-related methodologies between the two studies (i.e., M-mode vs. the modified Simpson's biplane method).

The lack of associations between resting LV parameters and peak CRF in this study is perhaps, unsurprising. During exercise, there are marked changes resulting from the interactions between different body systems, and the interplay of all of these systems cannot be explained simply by their resting state. The sympathetic arm of the autonomic nervous system plays a crucial role in bodily system adjustment in response to physiological stimuli (e.g., exercise, postural challenges). In non-injured individuals, the sympathetically induced vasoconstriction of peripheral vascular beds (splanchnic circulatory bed and lower limbs) and the action of skeletal muscle pumps serve to achieve appropriate venous return and increased end-diastolic ventricular volume (pre-load). As a result, in accordance with the Frank-Starling mechanism, SV is maintained. It might be interesting to speculate that these physiological responses apply to individuals with SCI. However, individuals with injuries $\geq \mathrm{T} 6$ experience drastic alterations to these normal physiological mechanisms, along with potentially reduced circulating catecholamines [9], which impact cardiac mechanics and exercise performance. Moreover, the reduced hemodynamic load induced by upper-extremity exercise alone may hinder these mechanisms. In the same context, the lower HR that is frequently observed due to sympathetic decentralization [9] might also result in reduced exercise-related stimuli. Both oxygen transport and extraction mechanisms are affected by the NLI and severity of injury [32]. It should be considered that the aforementioned central exercise-induced mechanisms do not account for the oxygen extraction (peripheral, i.e., skeletal muscle oxidative capacity) that might also be responsible for reduced CRF in individuals with SCI [33].

The cardiovascular system has mainly evolved for the efficient distribution of food/nutrients (e.g., the acquisition and distribution of oxygen) to metabolically active tissues, such as muscles, during an exercise stressor rather than to 
less metabolically active tissue (i.e., adipose) [22]. Compared to other body composition parameters, FFM represents the body compartments that dominate metabolic demands [34]. Previous studies in non-athletic and athletic adults have shown that FFM is associated with LVM and explains up to $30-37 \%$ of the variance in $\operatorname{LVM}[19,20]$. Our data are in accord with these findings and suggest that among cervical and upper-thoracic SCI, FFM is more strongly associated with LVM than FM, explaining 38\% of the variance. Theoretically, this might be useful in the indexing process, as it will allow for distinguishing between metabolically active tissues and those that are less metabolically active.

\section{Limitations}

This study is limited by its cross-sectional design. Thus, further studies are needed to investigate longitudinal changes in CRF and LV parameters. Exercise itself produces marked changes in LV performance; thus, future studies may want to investigate the association between exercise parameters and stress echocardiographic-obtained LV dynamic measures, preferably with the position in which the exercise was performed (i.e., upright or supine). DXA, among other techniques, is the current "gold standard" in determining body composition; however, it is less available in daily clinical practice. Devices such as a bioelectrical impedance analysis may offer a portable and alternative way of measuring body composition [22]. Due to the relatively small sample size, we were unable to perform a multivariate linear regression. Furthermore, the homogeneity and lower CRF levels of the included cohort (i.e., less variability) may impede our ability to detect large or significant associations [35]. Hence, larger studies with more heterogeneous samples are needed to draw more definitive conclusions.

\section{Conclusion}

In conclusion, resting LV parameters in individuals with cervical and upper-thoracic SCI are not associated with peak CRF. Nevertheless, assessing CRF is crucial to quantify the responses to exercise training, irrespective of LV parameters and likely provides an indication of peripheral adaptations and functional improvements. The nature of the associations between LVM and FFM needs further studies to investigate the possibility of using this measure to index LV parameters in the SCI population. Likewise, the association between parameters of CRF and body dimensions needs further investigation to determine the appropriate indexing approaches relative to active musculature (i.e., upper-body FFM).

\section{Data availability}

The data sets that were collected and analyzed for the purpose of this study are available from the corresponding author upon a reasonable request.

Acknowledgements We acknowledge the support of a Canadian Institutes of Health Research (CIHR) project grant (funding reference number; TCA 118348), the primary funding source for the Cardiovascular Health/Outcomes: Improvements Created by Exercise and education in SCI (CHOICES) multicenter project. We also thank the Canada Foundation for Innovation/BC Knowledge Translation Fund (funding reference number; 24906) for funding all equipment required for this study. We also thank the Craig $\mathrm{H}$. Neilson Foundation for funding the assessment of left ventricular structure/function (funding reference number; H13-03072). The authors would like to thank the participants of this study and study coordinators (Cameron Gee, Marko Gavric, Andrea Maharaj and Martina Franz) who were crucial for the success of the present study. AVK holds the Endowed Chair in Rehabilitation Medicine. The authors also acknowledge Dr Kathleen A. Martin Ginis's contribution to the selection of study measures for assessing self-reported physical activity. AAA is a recipient of $\mathrm{PhD}$ scholarship from King Fahad Medical City, Riyadh, Saudi Arabia. SJTB is a recipient of the Robert H.N. Ho Scholarship and UBC President's Academic Excellence Award. KDC was funded by Postdoctoral Fellowship from the Heart and Stroke Foundation (17R26776) and the Craig H. Neilson Foundation (281863). TEN was funded by Michael Smith Foundation for Health Research, Rick Hansen Foundation and ICORD Research Trainee Award. We also wish to acknowledge the study DXA technologist Dr Danmei Liu.

Funding Canadian Institutes of Health Research (CIHR) project grant (funding reference number; TCA 118348), Canada Foundation for Innovation/BC Knowledge Translation Fund (funding reference number; 24906), and Craig H. Neilson Foundation (funding reference number; H13-03072).

Author contributions AAA was responsible for collecting and analyzing the data, interpreting the results, drafting the paper and figures/tables, and approving the final paper. SJTB was responsible for collecting and analyzing the data, interpreting the results, revising and approving the final paper. KDC was responsible for designing the study, preparing the ethics, collecting and analyzing the data, interpreting the results, revising and approving the final paper. TEN was responsible for collecting and analyzing the data, interpreting the results, revising and approving the final paper. AVK was responsible for designing the study, preparing the ethics, recruiting participants, interpreting the results, revising and approving the final paper.

\section{Compliance with ethical standards}

Conflict of interest The authors declare that they have no conflict of interest.

Ethical approval We certify that all applicable institutional and governmental regulations concerning the ethical use of human volunteers were followed during this research.

Publisher's note Springer Nature remains neutral with regard to jurisdictional claims in published maps and institutional affiliations. 
Open Access This article is licensed under a Creative Commons Attribution 4.0 International License, which permits use, sharing, adaptation, distribution and reproduction in any medium or format, as long as you give appropriate credit to the original author(s) and the source, provide a link to the Creative Commons license, and indicate if changes were made. The images or other third party material in this article are included in the article's Creative Commons license, unless indicated otherwise in a credit line to the material. If material is not included in the article's Creative Commons license and your intended use is not permitted by statutory regulation or exceeds the permitted use, you will need to obtain permission directly from the copyright holder. To view a copy of this license, visit http://creativecommons. org/licenses/by/4.0/.

\section{References}

1. Ross R, Blair SN, Arena R, Church TS, Després JP, Franklin BA, et al. Importance of Assessing Cardiorespiratory Fitness in Clinical Practice: a Case for Fitness as a Clinical Vital Sign: a Scientific Statement From the American Heart Association. Circulation 2016;134:e653-e99.

2. Janssen TW, Dallmeijer AJ, Veeger DJ, van der Woude LH. Normative values and determinants of physical capacity in individuals with spinal cord injury. J Rehabil Res Dev. 2002;39:29-39.

3. Haisma JA, van der Woude LH, Stam HJ, Bergen MP, Sluis TA, Bussmann JB. Physical capacity in wheelchair-dependent persons with a spinal cord injury: a critical review of the literature. Spinal Cord. 2006; 44:642-52.

4. Simmons OL, Kressler J, Nash MS. Reference fitness values in the untrained spinal cord injury population. Arch Phys Med Rehabil. 2014;95:2272-8.

5. Noreau L, Shephard RJ. Spinal cord injury, exercise and quality of life. Sports Med. 1995;20:226-50.

6. Perhonen MA, Franco F, Lane LD, Buckey JC, Blomqvist CG, Zerwekh JE, et al. Cardiac atrophy after bed rest and spaceflight. J Appl Physiol (1985). 2001;91:645-53.

7. Williams AM, Gee CM, Voss C, West CR. Cardiac consequences of spinal cord injury: systematic review and meta-analysis. Heart. 2018;105:217-25.

8. Krassioukov AV, Bunge RP, Pucket WR, Bygrave MA. The changes in human spinal sympathetic preganglionic neurons after spinal cord injury. Spinal Cord. 1999;37:6-13.

9. Krassioukov A, West C. The role of autonomic function on sport performance in athletes with spinal cord injury. PM R. 2014;6(8 Suppl):S58-65.

10. Cragg JJ, Noonan VK, Krassioukov A, Borisoff J. Cardiovascular disease and spinal cord injury: results from a national population health survey. Neurology 2013;81:723-8.

11. Gardin JM, Lauer MS. Left ventricular hypertrophy: the next treatable, silent killer? JAMA 2004;292:2396-8.

12. Paulus WJ, Tschöpe C, Sanderson JE, Rusconi C, Flachskampf FA, Rademakers FE, et al. How to diagnose diastolic heart failure: a consensus statement on the diagnosis of heart failure with normal left ventricular ejection fraction by the Heart Failure and Echocardiography Associations of the European Society of Cardiology. Eur Heart J. 2007;28:2539-50.

13. Grewal J, McCully RB, Kane GC, Lam C, Pellikka PA. Left ventricular function and exercise capacity. JAMA 2009;301:286-94.

14. Brinker SK, Pandey A, Ayers CR, Barlow CE, DeFina LF, Willis BL, et al. Association of cardiorespiratory fitness with left ventricular remodeling and diastolic function: the Cooper Center Longitudinal Study. JACC Heart Fail. 2014;2: $238-46$.
15. Pandey A, Allen NB, Ayers C, Reis JP, Moreira HT, Sidney S, et al. Fitness in Young Adulthood and Long-Term Cardiac Structure and Function: the CARDIA Study. JACC Heart Fail. 2017;5: $347-55$.

16. Franciosa JA, Park M, Levine TB. Lack of correlation between exercise capacity and indexes of resting left ventricular performance in heart failure. Am J Cardiol. 1981;47:33-9.

17. Szlachcic J, Massie BM, Kramer BL, Topic N, Tubau J. Correlates and prognostic implication of exercise capacity in chronic congestive heart failure. Am J Cardiol. 1985;55:1037-42.

18. Okura $\mathrm{H}$, Inoue $\mathrm{H}$, Tomon $\mathrm{M}$, Nishiyama $\mathrm{S}$, Yoshikawa $\mathrm{T}$, Yoshida K, et al. Impact of Doppler-derived left ventricular diastolic performance on exercise capacity in normal individuals. Am Heart J. 2000;139:716-22.

19. Whalley GA, Gamble GD, Doughty RN, Culpan A, Plank L, MacMahon S, et al. Left ventricular mass correlates with fat-free mass but not fat mass in adults. J Hypertens. 1999;17: 569-74.

20. Whalley GA, Doughty RN, Gamble GD, Oxenham HC, Walsh HJ, Reid IR, et al. Association of fat-free mass and training status with left ventricular size and mass in endurance-trained athletes. J Am Coll Cardiol. 2004;44:892-6.

21. Daniels SR, Kimball TR, Morrison JA, Khoury P, Meyer RA. Indexing left ventricular mass to account for differences in body size in children and adolescents without cardiovascular disease. Am J Cardiol. 1995;76:699-701.

22. Dewey FE, Rosenthal D, Murphy DJ, Froelicher VF, Ashley EA. Does size matter? Clinical applications of scaling cardiac size and function for body size. Circulation 2008;117:2279-87.

23. Spungen AM, Adkins RH, Stewart CA, Wang J, Pierson RN, Waters RL, et al. Factors influencing body composition in persons with spinal cord injury: a cross-sectional study. J Appl Physiol (1985). 2003;95:2398-407.

24. Krassioukov AV, Currie KD, Hubli M, Nightingale TE, Alrashidi AA, Ramer L, et al. Effects of exercise interventions on cardiovascular health in individuals with chronic, motor complete spinal cord injury: protocol for a randomised controlled trial [Cardiovascular Health/Outcomes: Improvements Created by Exercise and education in SCI (CHOICES) Study]. BMJ Open. 2019;9: e023540.

25. Martin Ginis KA, Úbeda-Colomer J, Alrashidi AA, Nightingale TE, Au JS, Currie KD, et al. Construct validation of the leisure time physical activity questionnaire for people with SCI (LTPAQSCI). Spinal Cord. 2020. https://doi.org/10.1038/s41393-02000562-9.

26. Kirshblum SC, Burns SP, Biering-Sorensen F, Donovan W, Graves DE, Jha A, et al. International standards for neurological classification of spinal cord injury (revised 2011). J Spinal Cord Med. 2011;34:535-46.

27. Lang RM, Badano LP, Mor-Avi V, Afilalo J, Armstrong A, Ernande L, et al. Recommendations for cardiac chamber quantification by echocardiography in adults: an update from the American Society of Echocardiography and the European Association of Cardiovascular Imaging. J Am Soc Echocardiogr. 2015;28:1-39.e14.

28. Devereux RB, Alonso DR, Lutas EM, Gottlieb GJ, Campo E, Sachs I, et al. Echocardiographic assessment of left ventricular hypertrophy: comparison to necropsy findings. Am J Cardiol. 1986;57:450-8.

29. Eerden S, Dekker R, Hettinga FJ. Maximal and submaximal aerobic tests for wheelchair-dependent persons with spinal cord injury: a systematic review to summarize and identify useful applications for clinical rehabilitation. Disabil Rehabil. 2018;40:497-521.

30. Maggioni MA, Ferratini M, Pezzano A, Heyman JE, Agnello L, Veicsteinas A, et al. Heart adaptations to long-term aerobic 
training in paraplegic subjects: an echocardiographic study. Spinal Cord. 2012;50:538-42.

31. West CR, Campbell IG, Shave RE, Romer LM. Effects of abdominal binding on cardiorespiratory function in cervical spinal cord injury. Respir Physiol Neurobiol. 2012;180: 275-82.

32. Theisen D. Cardiovascular determinants of exercise capacity in the Paralympic athlete with spinal cord injury. Exp Physiol. 2012;97:319-24.
33. Hopman MT, Dueck C, Monroe M, Philips WT, Skinner JS. Limits to maximal performance in individuals with spinal cord injury. Int J Sports Med. 1998;19:98-103.

34. Savage DD, Levy D, Dannenberg AL, Garrison RJ, Castelli WP. Association of echocardiographic left ventricular mass with body size, blood pressure and physical activity (the Framingham Study). Am J Cardiol. 1990;65:371-6.

35. Goodwin LD, Leech NL. Understanding Correlation: factors That Affect the Size of r. J Exp Educ. 2006;74:251-66. 